\title{
AN ATLAS OF EXTRAGALACTIC RADIO SOURCES
}

\author{
J.P. LEAHY ${ }^{1}$, A.H. BRIDLE ${ }^{2}$, R.G. STROM ${ }^{3}$ \\ 1 University of Manchester - Jodrell Bank, Cheshire, UK \\ ${ }^{2}$ N.R.A.O. - Charlottesville, Virginia 22903, USA \\ ${ }^{3}$ N.F.R.A. - PO Box 2, 7990 AA Dwingeloo, the Netherlands
}

\section{Overview}

Our Atlas of Extragalactic Radio Sources will present high-quality images of the nearer half of "3CRR", the sample defined by Laing, Riley \& Longair (1983). This is the best-studied complete sample of extragalactic radio sources. All 173 members have secure redshifts and most have been imaged in the radio at high resolution. There is also copious information on their optical line emission, and many have been detected in the sub-mm, FIR, and in X-rays. 3CRR is widely used as a baseline against which fainter, higher-redshift samples can be compared to define the evolution of the population (e.g. Neeser et al. 1995; Law-Green, this conference).

At present, modern radio data on 3CRR is partly unpublished and the rest is spread across many papers. The digital images are not easily accessible, which hinders systematic morphological analysis. The Atlas will solve (half) this problem. Many of its images have been contributed by other observers, to whom we are very grateful.

The sample is defined as: extragalactic objects with $S_{178 \mathrm{MHz}} \geq 10.9$ $\mathrm{Jy}, \delta \geq 10^{\circ}$ and $b^{I I} \geq 10^{\circ}$ (these define 3CRR), and $z \leq 0.5$; we exclude the starburst galaxy 3C 231 (M 82), to give 85 members, all DRAGNs. The high-redshift part of 3CRR is excluded mainly because the large majority have been imaged at high resolution by Laing \& Owen (unpublished).

The sample contains 21 objects with powers below the FR break, and of these only three "fat doubles" lack jets. About a third of the objects above the FR break differ from the "classical" symmetric structure with hotspots at both ends. Twenty-seven of the 59 powerful radio galaxies show jets; another 6 have possible jets. Of these, 12 (possibly 13) have twin jets, 


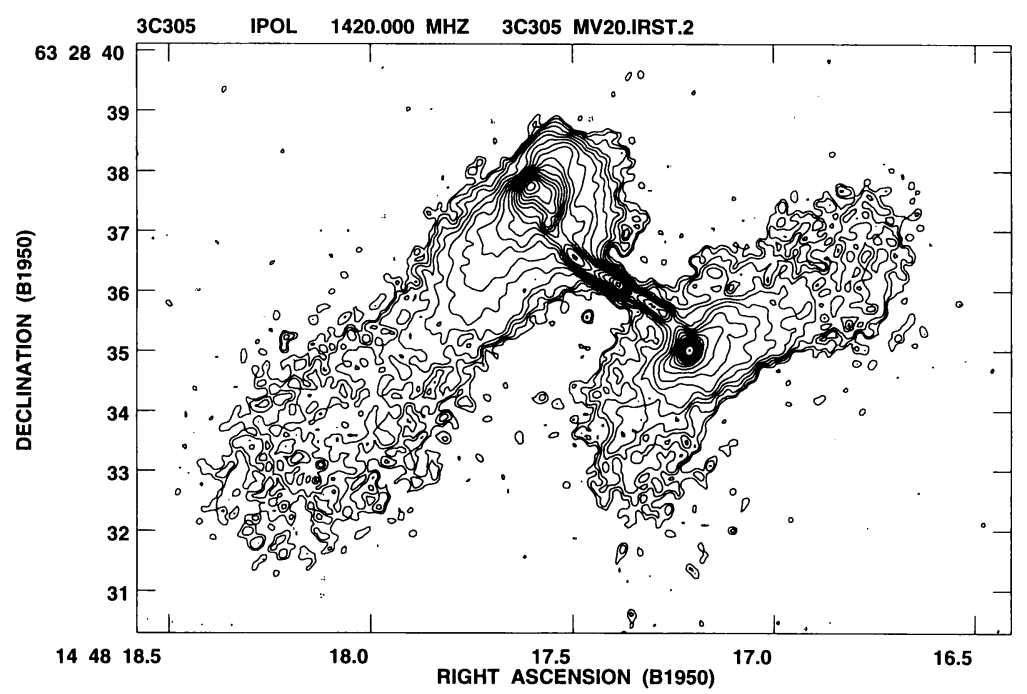

Figure 1. 3C 305 at $20 \mathrm{~cm}$ (MERLIN + VLA image). Beam 0.15 arcsec.

suggesting that jets are much less one-sided in powerful radio galaxies than quasars; however, only 4 (5) of these are classical doubles. There are also 5 quasars, all with jets. Cores are now detected in nearly all sample members (the exceptions have usually not been observed to sub-mJy levels at high frequency). Not all these features are visible in the Atlas images.

The Atlas images have FWHM beam $\leq 5 \%$ of the target largest angular size (typically $\sim 2.5 \%$.), and sensitivity and uv-coverage sufficient to show the largest and faintest components in each DRAGN. The images are mostly at $20 \mathrm{~cm}$, and are from the WSRT, VLA, MERLIN or EVN as appropriate for the angular size. The Atlas will also contain tables of basic data on each DRAGN, including flux densities from $10 \mathrm{MHz}$ to $1 \mathrm{keV}$, basic morphological data derived from the images, and possibly optical spectra.

We now have images for nearly all sample members, although in a few cases they do not meet (or barely meet) our target criteria, and improved images are being produced. We hope to make our current collection of images available on the World-Wide Web (look for details on http://wwr.jb.man.ac.uk/jb.html). Distribution on CD should occur in 1996 as part of the NRAO CD series; publication in print may be some time later. Fig. 1 shows an image recently made for the project.

\section{References}

Laing, R.A., Riley, J.M., Longair, M.S., 1983. MNRAS, 204, 151.

Law-Green, J.D.B. et al. 1995. MNRAS, 274, 939.

Neeser, M.J, Eales, S.A., Law-Green, J.D., Leahy, J.P., Rawlings, S. 1995. ApJ, 451, 76. 